\title{
Effect of Added Surfactant on Poly(Ethylenimine)-Assisted Gold Nanoparticle Formation
}

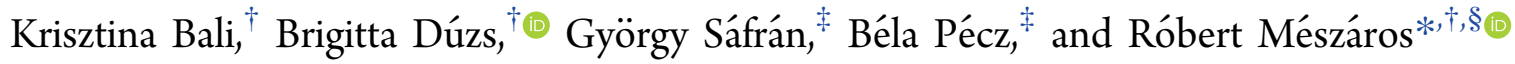 \\ ${ }^{\dagger}$ Laboratory of Interfaces and Nanosized Systems, Institute of Chemistry, ELTE Eötvös Loránd University, H-1117 Budapest, \\ Pázmány Péter sétány $1 / \mathrm{A}$, Hungary \\ ${ }^{\ddagger}$ Institute of Technical Physics and Materials Sciences, Centre for Energy Research, H.A.S., H-1121 Budapest, Konkoly Thege M. út \\ 29-33, Hungary \\ ${ }^{\S}$ Department of Chemistry, University J. Selyeho, 94501 Komárno, Slovakia
}

Supporting Information

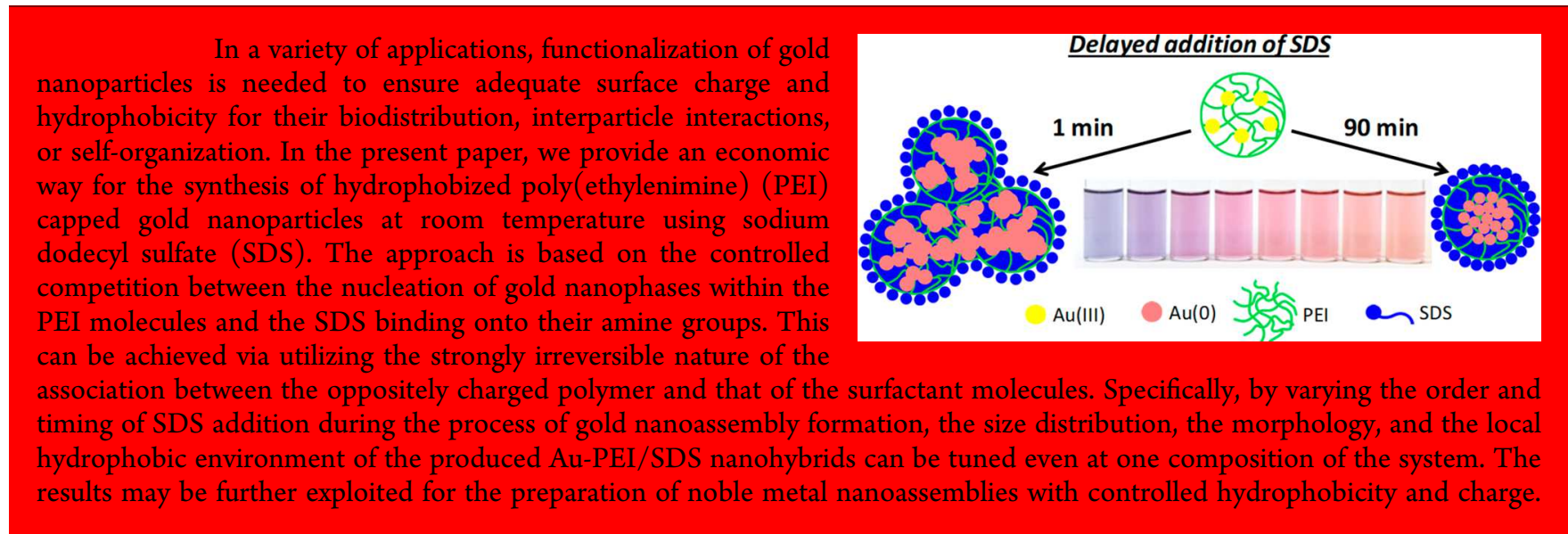

\section{INTRODUCTION}

The preparation and functionalization of gold nanoparticles (AuNPs) have initiated intensive research in the last two decades since they represent great potential in a variety of fields such as catalysis, optical, and spectroscopic devices as well as biomedical formulations and colorimetric sensors. ${ }^{1-4}$ The various applications utilize a diversity of different gold nanosystems with specific requirements for the size, shape, stability, and hydrophobicity of these AuNPs. The synthesis is usually based on the reduction of gold(III) ions followed by the controlled growth of NPs with stabilizing additives., 5 Nowadays, a great deal of different preparation methods is routinely used, both in aqueous and nonpolar media. An important current requirement is related to green nanoparticle synthesis routes, i.e., excluding high temperature conditions as well as excessive usage of organic solvents and additives.?

Polyelectrolytes (PE) play a crucial role in the stabilization and applications of AuNPs via their controlled interactions with the preformed particles. ${ }^{8-12}$ Among the different polyelectrolytes, polyamines offer a unique alternative for gold NP synthesis since they may simultaneously act as reducing, stabilizing, and capping agent as well. In particular, branched polyethylenimines (PEI) are frequently utilized for gold NP synthesis due to their commercial availability, biocompatibility, and dendrimer-like structure. ${ }^{13-18}$ Depending on the applied solvent and experimental conditions, either single gold $\mathrm{NPs}^{16,19}$ or supraparticles embedded in the PEI matrix may be formed. ${ }^{17,20,21}$

Recent studies revealed the optimization of PEI-assisted gold nanoparticle synthesis ${ }^{22}$ and their extensive applications. ${ }^{22-27}$ For instance, Philip et al. synthesized large AuNPs for surfaceenhanced Raman scattering studies via a PEI-assisted seedmediated method, where the size and polydispersity of the NPs were controlled by the ratio of the PEI and that of the seeds. ${ }^{26}$ PEI-capped AuNPs have also been suggested for medical applications $^{23-25}$ as well as for colorimetric sensing of heparin $^{27}$ due to the good stability of their dispersions in the biologically relevant $\mathrm{pH}$ and temperature range. Kim et al. revealed that the PEI entrapped AuNPs could be used for the preparation of specific surface films at the water-hexane interface in the presence of alcohol containing alkanethiols. ${ }^{28}$

In the majority of the applications of gold NPs, their further functionalization is of paramount importance. For instance, the surface charge and hydrophobicity of the NPs have a prominent role in the biodistribution and self-organization of AuNPs in aqueous medium. ${ }^{29,30}$ For this aim, Mitamura et al. investigated the self-assembly of cetyltrimethylammonium bromide (CTAB) coated AuNPs in the presence of other additives. However, for the fabrication of a hydrophobic shell around the AuNPs, a three step method was needed by making

Received: September 30, 2019

Published: October 7, 2019 

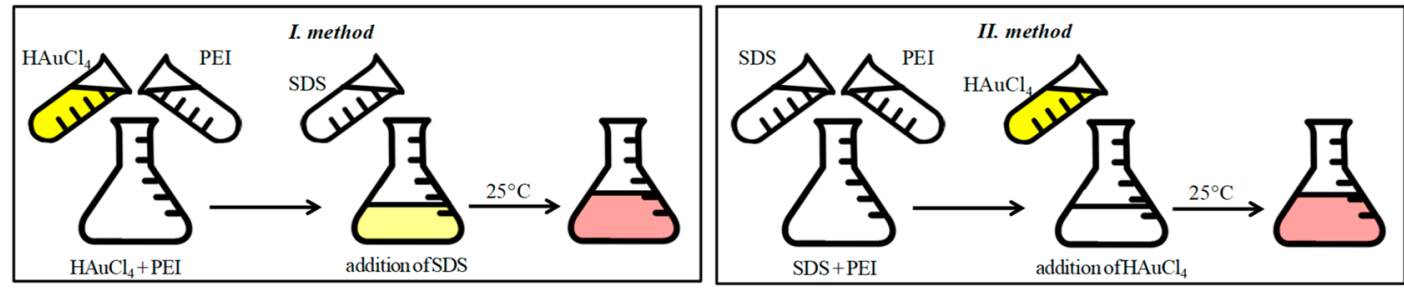

Figure 1. Schematic illustration of the I and II sample preparation methods. After their preparation, the samples were stored at $25{ }^{\circ} \mathrm{C}$ before measurements.

use of silanes and organic solvents as well. ${ }^{31}$ In principle, polyelectrolyte/surfactant (PE/S) interactions can also be used for surface modification and functionalization. For instance, chemically $^{12}$ or physically bound ${ }^{10}$ surfactant layers were used earlier to fabricate alternating polyelectrolyte layers. However, to ensure adequate charge of these surfactant coated gold NPs, usually multistep methods and/or chemical functionalization of the gold NPs are needed. Furthermore, although these multilayers may be used for interesting applications, such as the fabrication of hollow polyelectrolyte capsules, they are not necessarily suitable for the preparation of gold NPs with tunable hydrophobicity and charge.

In our previous work, it was shown that the charge and hydrophobicity of the PEI-capped AuNPs, prepared at $80{ }^{\circ} \mathrm{C}$, can be modified via their rapid mixing with sodium alkyl sulfate surfactants due to their interaction with the remaining protonated amine groups of PEI. ${ }^{32}$ This is attributable to those earlier findings, which revealed that the charge, structure, and hydrophobic character of aqueous PEI-SDS complexes were largely dependent on the protonation degree of PEI as well as on the bound amount of SDS. ${ }^{33-38}$ These studies also indicated that the various properties of this system could be largely affected through the application of adequate solution preparation methods, due to the nonequilibrium nature of oppositely charged polyelectrolyte/surfactant association at low ionic strengths. ${ }^{33-35,39-42}$

In the present paper, we provide an economic and simple synthesis method of Au-PEI/SDS nanoassemblies with more versatile structure and size distribution than in the case of the high temperature method. ${ }^{32}$ For this aim, we will manipulate various processes, which occur simultaneously at room temperature and primarily governed by the available amine groups of PEI; i.e. the anionic surfactant binding, formation of gold complexes, the reduction of $\mathrm{Au}$ (III) ions, and the stabilization of the gold NPs. Specifically, the effect of the order of addition on the formation of gold nanoassemblies will be explored using dynamic light scattering (DLS), electrophoretic mobility, UV-vis-NIR, and fluorescent spectroscopy as well as TEM techniques. We will demonstrate that the controlled order and timing of SDS addition to PEI and $\mathrm{HAuCl}_{4}$ result in gold nanohybrids with tunable size, morphology, and hydrophobicity at the same analytical composition of the system.

\section{EXPERIMENTAL SECTION}

Materials. The sodium dodecyl sulfate sample (SDS, SigmaAldrich, $\geq 98.0 \%$ ) was recrystallized twice from ethanol. The $\mathrm{cmc}$ of SDS in water was found to be $8.1 \mathrm{mM}$ at $25.0 \pm 0.1{ }^{\circ} \mathrm{C}$ from conductivity measurements. The branched poly(ethylenimine) (PEI, Sigma-Aldrich) sample has a mass averaged molar mass of $25 \mathrm{kDa}$ with an approximate 1:2:1 ratio of the primary, secondary, and tertiary amine $_{\text {groups. }}{ }^{33}$ Ultrapure water (Milli-Q) was used for the preparation of the solutions.

Solution Preparation Methods. The aqueous solution of $\mathrm{HAuCl}_{4}$ was added to poly(ethylenimine) PEI solution to prepare a $20 \mathrm{~mL}$ mixture $\left(\mathrm{HAuCl}_{4}-\mathrm{PEI}\right.$ premix) under continuous stirring with a magnetic stirrer $(1800 \mathrm{rpm})$. The final PEI (in ethylenimine (EI) monomer concentration) and $\mathrm{HAuCl}_{4}$ concentrations were 1.2 and $0.2 \mathrm{mM}$, respectively, which resulted in a mixture with $\mathrm{pH} 4$ (measured by a ProLab $1000 \mathrm{pH}$ meter using a combined glass electrode). The SDS stock solution was prepared freshly and used within 1 day. In our experiments, three different sample preparation methods were used as described below.

I. Method: $\mathrm{HAuCl}_{4}+\mathrm{PEI} \rightarrow$ Addition of SDS. During the first sample preparation method, $1.5 \mathrm{~mL}$ SDS solution was added rapidly to the $1.5 \mathrm{~mL} \mathrm{HAuCl}_{4}$-PEI premix (immediately after its preparation) at room temperature under continuous stirring (1800 $\mathrm{rpm})$. The mixtures were stored at $25{ }^{\circ} \mathrm{C}$ before measurements (usually 1 day and/or 1 week after preparation).

II. Method: SDS + PEI $\rightarrow$ Addition of $\mathrm{HAuCl}_{4}$. At first, $1.44 \mathrm{~mL}$ PEI solution and $1.44 \mathrm{~mL}$ SDS solution were mixed by a magnetic stirrer (1800 rpm). Next, $0.12 \mathrm{~mL}$ concentrated $\mathrm{HAuCl}_{4}$ solution was added immediately to the PEI/SDS mixture under continuous stirring (1800 $\mathrm{rpm}$ ) to get the same final concentrations as via the application of the I method. The prepared mixtures were stored at $25{ }^{\circ} \mathrm{C}$ before measurements. The illustration of the first two preparation methods (I and II) is shown in Figure 1.

III. Method: $\mathrm{HAuCl}_{4}+\mathrm{PEI} \rightarrow$ Delayed Addition of SDS. This mixing protocol is the extension of the I method. However, the 1.5 $\mathrm{mL}$ SDS solution was added to the $1.5 \mathrm{~mL} \mathrm{HAuCl}_{4}$-PEI premix (under continuous stirring at $1800 \mathrm{rpm}$ ) only after a well-defined waiting time period, which is called delay time $\left(t_{\mathrm{D}}\right)$ throughout the text which usually varied between 1 and 90 min (occasionally much longer delay times are also applied). After this delayed surfactant addition, the mixtures were kept at $25{ }^{\circ} \mathrm{C}$ before measurements. The illustration of this preparation method is shown in Figure 2.

UV-vis-NIR Spectroscopy. The formation of AuNPs was monitored by observing changes in the absorption spectra at $25^{\circ} \mathrm{C}$.

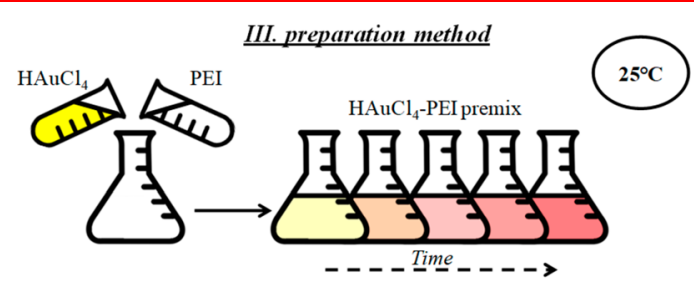

Delav time before SDS addition

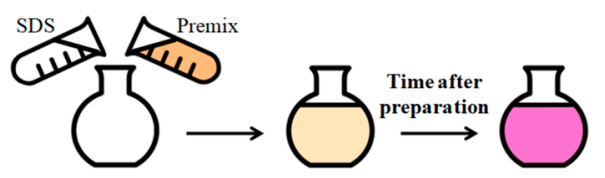

Figure 2. Schematic illustration of the III preparation method (delayed addition of SDS). 

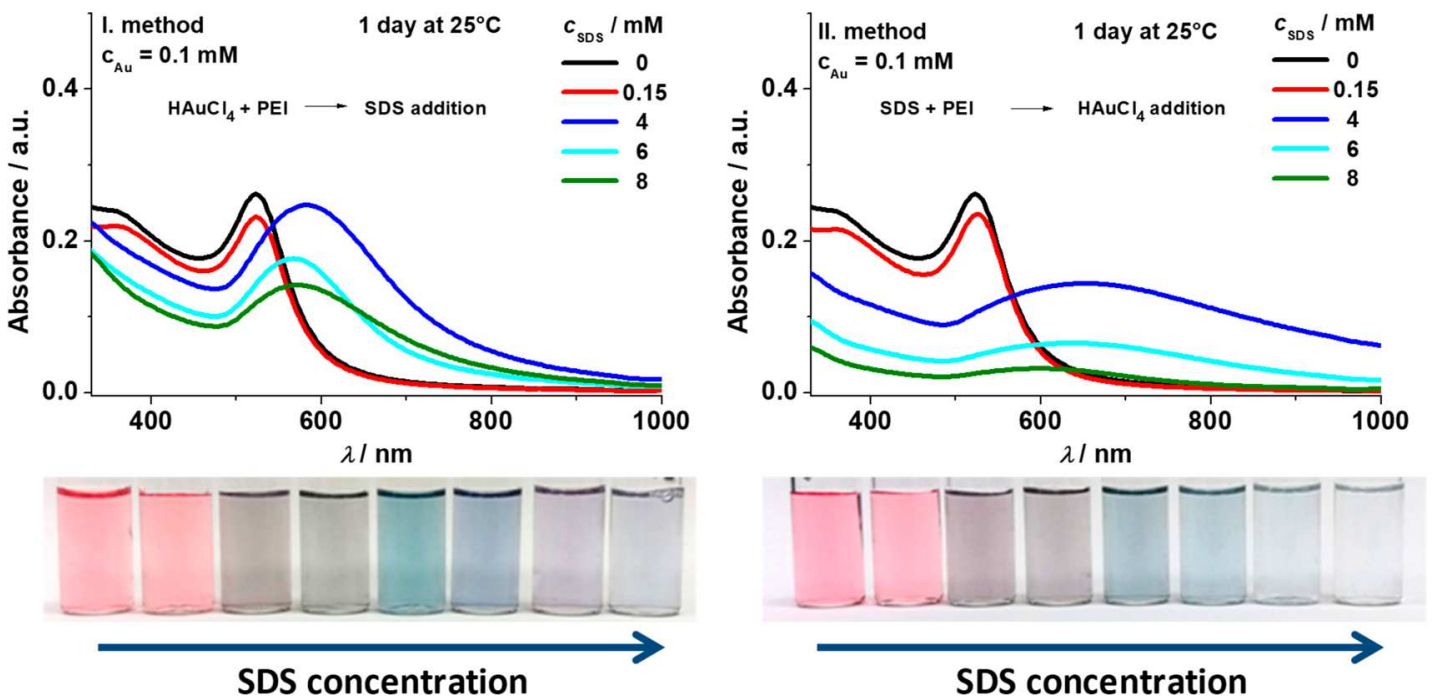

Figure 3. UV-vis-NIR spectra and photos of the samples 1 day after their preparation by the I (left) and the II (right) methods. The width of the lines is commensurable with the standard error of the measurements. On the photos, the SDS concentration increases from left to right $(0 ; 0.15$; $0.7 ; 1 ; 2 ; 4 ; 6 ; 8 \mathrm{mM}$, respectively). $c_{\mathrm{Au}}=0.1 \mathrm{mM}$ and $c_{\mathrm{PEI}}=0.6 \mathrm{mM}$.

A PerkinElmer Lambda 1050 UV-vis-NIR spectrophotometer was used to record the spectra in the $200-1000 \mathrm{~nm}$ wavelength interval in a quartz cuvette with path length of $1.00 \mathrm{~cm}$. In some cases, the spectra are shown in the $320-800 \mathrm{~nm}$ wavelength range for the sake of simplicity.

Electrophoretic Mobility Measurements. The mean electrophoretic mobility $\left(u_{\zeta}\right)$ of the gold/PEI/SDS nanoassemblies was determined at $25.0 \pm 0.1{ }^{\circ} \mathrm{C}$, using a Malvern Zetasizer Nano ZSP instrument. The apparatus utilizes the M3-PALS technique to derive the mean velocity of the nanoassemblies $\left(v_{E}\right)$ at a given electric field strength $(E)$, from the measured frequency shift of the scattered light due to the moving particles. The mean mobility values are given from the well-known $u_{\zeta}=v_{E} / E$ relationship.

Dynamic Light Scattering (DLS) Measurements. The mean size of the nanoassemblies was monitored by dynamic light scattering. The experimental setup (Brookhaven Instruments) consisted of a BI200SM goniometer system and a BI-9000 AT digital correlator using a Genesis MX488-1000 OPS laser (1 W). The measurements were carried out at $\lambda=488 \mathrm{~nm}$ wavelength, at $\theta=90^{\circ}$ scattering angle and at $25.0 \pm 0.1{ }^{\circ} \mathrm{C}, 24 \mathrm{~h}$ as well as 1 week after the preparation of the systems. The mixtures were filtered through a $0.45 \mu \mathrm{m}$ pore-size membrane filter prior to the measurements. The apparent mean diffusion coefficient of the particles $\left(D_{\text {app }}\right)$ was derived from the second-order cumulant analysis of the autocorrelation functions and their apparent mean hydrodynamic diameter $\left(d_{\mathrm{H}}\right)$ was calculated on the basis of the Einstein-Stokes relation: $D_{\text {app }}=\frac{k_{\mathrm{B}} T}{3 \pi \eta d_{\mathrm{H}}}$ where $T$ is the temperature, $k_{\mathrm{B}}$ is the Boltzmann constant, $\eta$ is the viscosity of the medium. Occasionally, the apparent mean Stokes diameters were also determined from DLS measurements taken at $\theta=173^{\circ}$ scattering angle by the back scattering utility of the previously described Malvern Zetasizer Nano ZSP instrument using a $10 \mathrm{~mW} \mathrm{He}-\mathrm{Ne}$ laser at $\lambda=633 \mathrm{~nm}$. In the investigated concentration range, the $d_{\mathrm{H}}$ values were not dependent within experimental errors on the type of the applied DLS setups.

Transmission Electron Microscopy (TEM). The nanoparticle dispersions were drop-dried on carbon coated microgrids for the TEM study. A Philips CM $20(200 \mathrm{kV})$ microscope was used for the conventional electron microscopy (bright field and dark field images) in order to characterize the shape and average size of the particles.

Steady-State Fluorescent Measurements. An appropriate amount of pyrene was dissolved in acetone and this solution was placed into a set of vials from which the solvent was removed. Then the given aqueous mixtures were poured into these vials. The pyrene concentration was $1 \times 10^{-6} \mathrm{M}$ in each sample. Before the fluorescence measurements, the given systems were stirred for $24 \mathrm{~h}$. Various $\mathrm{HAuCl}_{4} / \mathrm{PEI} / \mathrm{SDS}$ mixtures were prepared with one of the mixing methods described above. After their preparation, the systems were thermostated for a day or for a week before putting them into the pyrene containing vials. Control experiments were also performed with SDS, PEI/SDS, and PEI solutions as well as with surfactant free $\mathrm{Au}$-PEI nanoparticle dispersions. Pyrene fluorescent spectra were measured on a JASCO FP-8300-LC fluorimeter. The excitation wavelength was $320 \mathrm{~nm}$ and emissions were recorded in the 360-420 $\mathrm{nm}$ wavelength range. The characteristic $I_{1} / I_{3}$ ratio of the fluorescence intensities belonging to the first $(372 \mathrm{~nm})$ and third $(385 \mathrm{~nm})$ vibronic bands was used to monitor the micropolarity of the formed nanoassemblies. To test reproducibility, all of the systems were prepared five times by a given preparation method (i.e., by the II method or by the III method at the chosen delay times). The presented $I_{1} / I_{3}$ ratios are the averages of those values measured for the five samples.

\section{RESULTS AND DISCUSSION}

We start our discussion with the comparison of the impact of the first two preparation methods on the gold nanoassembly formation. In Figure 3, the UV-vis-NIR spectra of the mixtures 1 day after their preparation via the $\mathrm{I}\left(\mathrm{HAuCl}_{4}+\mathrm{PEI}\right.$ $\rightarrow$ addition of SDS: left panel) and the II (SDS + PEI $\rightarrow$ addition of $\mathrm{HAuCl}_{4}$ : right panel) methods are shown at different surfactant concentrations, which are outside of the precipitation regime.

The application of the smallest surfactant concentration results in a very similar surface plasmon resonance (SPR) peak of the spectrum as that of the quasispherical bare gold NPs, for both mixing methods. In contrast, at higher SDS concentrations both the spectra and the visual appearance of the samples (as indicated by the photos at the bottom of Figure 3) are largely different compared to the surfactant free, PEI capped gold NPs. Furthermore, they are also significantly dependent on the preparation protocols. Specifically, at surfactant excess the SPR peaks are wider and there is a red shift in the peak positions for the samples prepared by the I method as compared to the bare gold NPs. The application of the II method also leads to a red shift of the SPR peak; however, the peak becomes even wider alluding to the presence of large aggregates. Furthermore, at the highest 
investigated surfactant concentrations (e.g., at 6 and $8 \mathrm{mM}$ SDS) almost colorless samples with low absorbance values as well as the absence of an SPR peak was observed for the second preparation method. As shown, in Figure S1, in the case of the first preparation method, the peak positions did not change remarkably, whereas the absorbance values and the color intensity are enhanced considerably within 1 week, especially at surfactant excess. On the other hand, 7 days after the preparation of the samples by the II mixing method, wide SPR peaks as well as more intense bluish colors and enhanced absorbance values are observable even at the highest SDS concentrations, indicating the formation of large gold NP aggregates.

In Figure 4, the mean electrophoretic mobility $\left(u_{\zeta}\right)$ and the apparent mean Stokes diameter $\left(d_{\mathrm{H}}\right)$ of the gold nano-

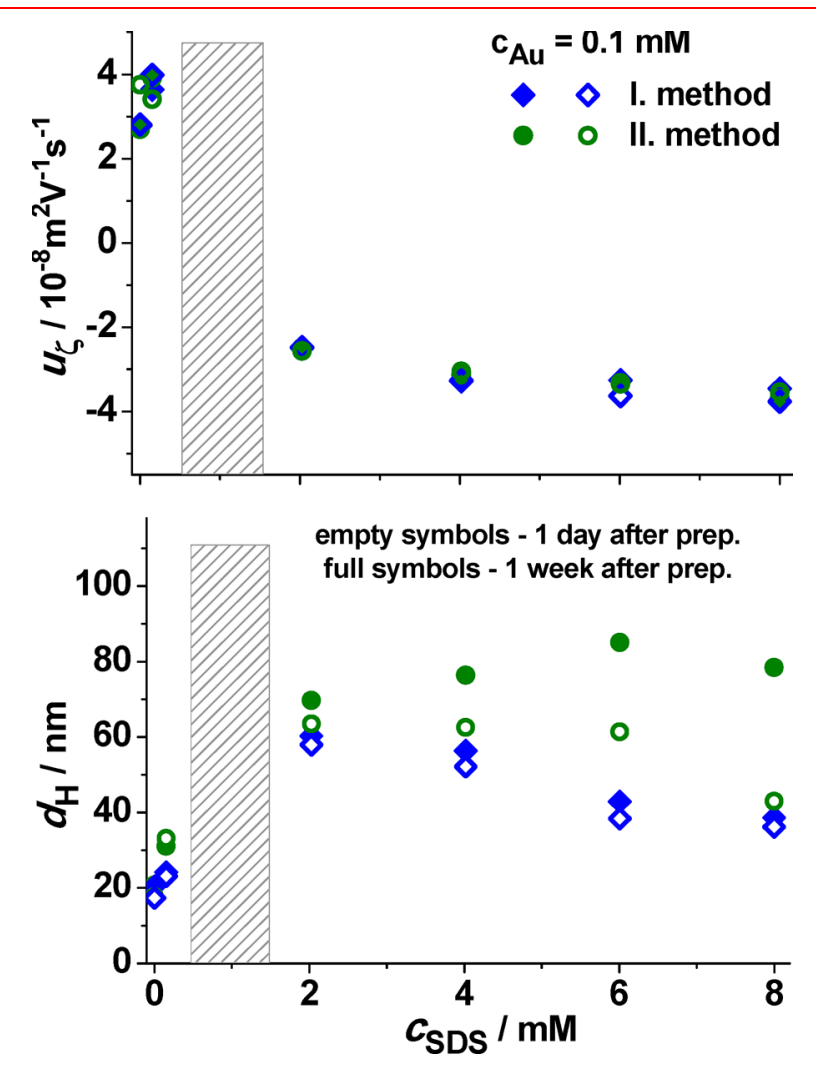

Figure 4. Mean electrophoretic mobility and the apparent mean diameter of the gold nanoassemblies against the surfactant concentration for the samples made by the I (blue diamonds) and the II (green circles) mixing methods, 1 day (empty symbols) and 1 week (full symbols) after their preparation. The gray rectangle indicates the precipitation region. $c_{\mathrm{Au}}=0.1 \mathrm{mM}, c_{\mathrm{PEI}}=0.6 \mathrm{mM}$.

assemblies are plotted 1 day and 1 week after their preparation, respectively, via the first and second mixing protocols. The bare, PEI capped gold NPs form a stable dispersion due to the electrostatic stabilization of the remaining protonated amine groups, which did not participate in the reduction process of $\mathrm{Au}(\mathrm{III})$ ions. The association with SDS compensates the positive charges of the PEI capped AuNPs, which leads to their coagulation and precipitation at intermediate surfactant concentrations. With a further increase of the SDS concentration, however, dispersions of negatively charged gold nanohybrids are formed.

A remarkable feature of Figure 4 that the mobility values of the $\mathrm{Au}-\mathrm{PEI} / \mathrm{SDS}$ nanoassemblies are dependent neither on the order of addition of SDS, nor the time passed after their preparation. In contrast, the mean size of the NPs and its time dependence reveal large deviations for the two mixing methods, especially at surfactant excess. Specifically, during 1 week, significant aggregation occurs in the case of the second preparation protocol, whereas the mean diameter of the gold nanoassemblies does not change considerably in time for the first preparation method. As a result, after 1 week the mean size of the NPs formed through the application of the II method becomes significantly larger than that of the samples prepared by the I method. However, even via the application of the first method, the mean diameter of the overcharged nanoassemblies $\left(d_{\mathrm{H}} \approx 40 \mathrm{~nm}\right)$ is considerably larger compared to that of the bare PEI entrapped AuNPs $\left(d_{\mathrm{H}} \approx 20 \mathrm{~nm}\right)$.

The large agglomerates of the gold nanoassemblies, prepared by the I and II methods at surfactant excess (between 2 and 8 mM SDS), are shown in the TEM images of Figure 5. As can

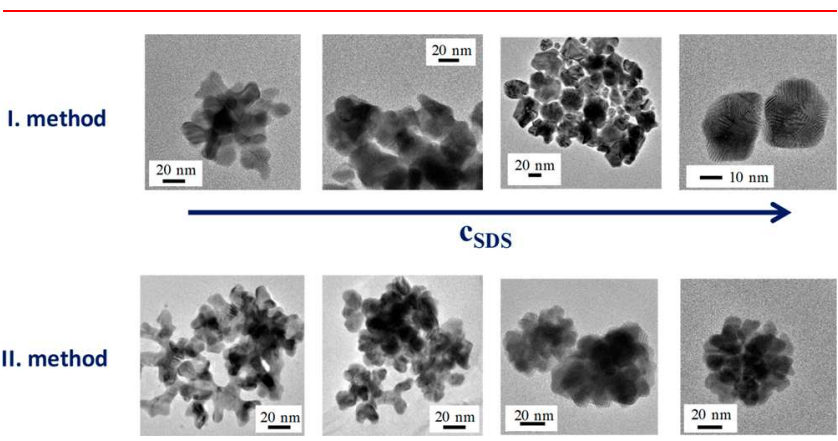

Figure 5. TEM images of Au PEI/SDS systems prepared via the I (top) and II (bottom) protocols 1 week after their preparation. The surfactant concentration increases from left to right such as $2,4,6$, and $8 \mathrm{mM}$.

be seen, in the case of the first method more compact and closed aggregates are formed as compared to the looser aggregate structures developed upon the application of the II method.

These observations are remarkably different compared with our earlier results on PEI-capped gold NPs, which were presynthesized at $80{ }^{\circ} \mathrm{C}$ and then mixed rapidly with sodium alkyl sulfate solutions. In that case, dispersions of overcharged gold nanohybrids with much smaller particle size $\left(d_{\mathrm{H}} \approx 25\right.$ $\mathrm{nm}$ ) and pronounced SPR peaks were produced. ${ }^{32}$

The reasons behind these deviations are primarily attributable to the temperature dependent mechanism of gold NP nucleation and growth as well as its interference with the surfactant binding and repartitioning processes. As we have shown previously, in the investigated PEI and $\mathrm{HAuCl}_{4}$ concentration range, the Au-PEI NP synthesis was completed within $2 \mathrm{~h}$ at $80{ }^{\circ} \mathrm{C} .{ }^{32}$ However, the reduction of $\mathrm{Au}(\mathrm{III})$ ions through the amine groups of PEI is a much slower process at $25^{\circ} \mathrm{C}$. This is illustrated in Figure 6a, through the variation of the $\mathrm{UV}$-vis spectrum of $\mathrm{HAuCl}_{4} / \mathrm{PEI}$ premix solution in time $\left(c_{\mathrm{Au}(\mathrm{III})}=0.2 \mathrm{mM}, c_{\mathrm{PEI}}=1.2 \mathrm{mM}\right)$ after its preparation together with the time independent spectrum of the aqueous $\mathrm{HAuCl}_{4}$ solution. The time dependence of the spectrum, measured at $0.1 \mathrm{mM} \mathrm{Au}(\mathrm{III})$ and $0.6 \mathrm{mM}$ PEI (which are the final concentrations of these components in the $\mathrm{Au} / \mathrm{PEI} / \mathrm{SDS}$ mixtures), is shown in Figure S2 of the SI). As shown in Figure $6 \mathrm{a}$, the polymer free aqueous gold(III) complex (at $\mathrm{pH} 4$ ) can be characterized with a pronounced absorption peak at approximately $220 \mathrm{~nm}$, and a slight shoulder at $274 \mathrm{~nm}$. 
a.)

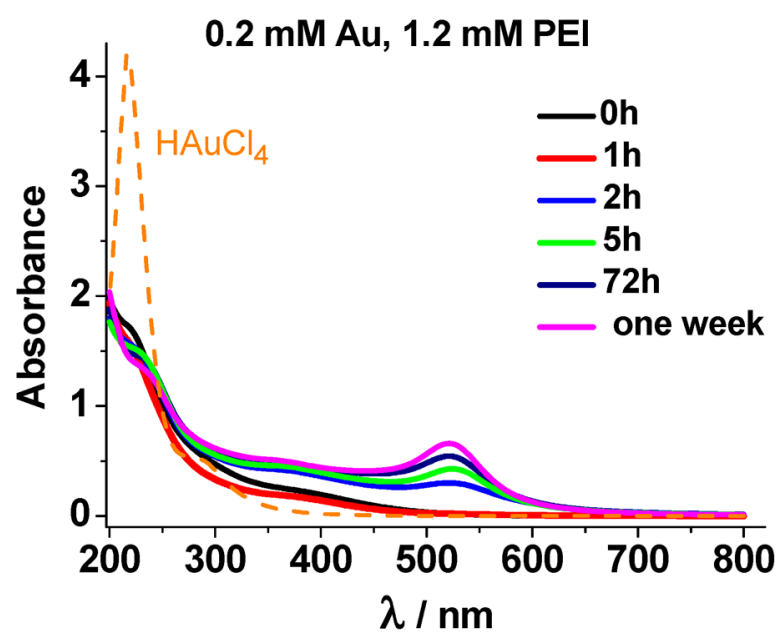

b.)

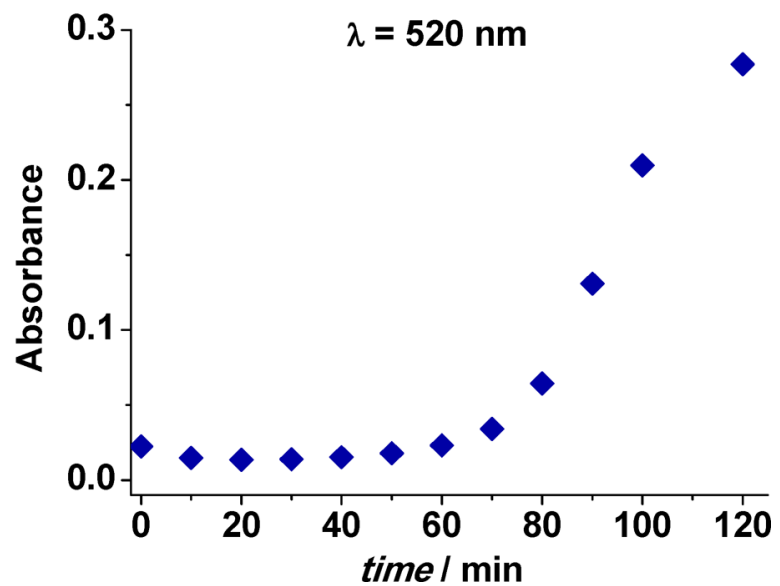

Figure 6. Time dependence of the (a) UV-vis spectra and (b) that of the absorbance measured at $\lambda=520 \mathrm{~nm}$ directly after the preparation of the $\mathrm{HAuCl}_{4}-\mathrm{PEI}$ mixture $\left(c_{\mathrm{Au}}=0.2 \mathrm{mM}, c_{\mathrm{PEI}}=1.2 \mathrm{mM}\right)$ at $t=25$ ${ }^{\circ} \mathrm{C}$.

This is due to the formation of $\mathrm{Au}(\mathrm{III})\left(\mathrm{Cl}^{-}\right)_{x}\left(\mathrm{OH}^{-}\right)_{y}$ complexes ${ }^{43}$ with $\mathrm{pH}$ dependent values of $x$ and $y(x \approx 2.46$ and $y \approx 1.54$ at $\mathrm{pH}=4$ ). According to Figure $6 \mathrm{a}$ and S2, however, the mixed chloro/hydroxyl complex is converted instantaneously to a gold(III)/polyamine complex, ${ }^{20,25}$ which is also indicated visually by the appearance of a deep yellow color upon mixing PEI with $\mathrm{HAuCl}_{4}$. Then, the amount of $\mathrm{Au}(\mathrm{III}) / \mathrm{PEI}$ complex slowly reduces with a simultaneous increase in the amount of gold NPs with a characteristic surface plasmon resonance (SPR) peak at $520 \mathrm{~nm}$ and a mild shoulder in the UV region at $226 \mathrm{~nm}$.

In Figure $6 \mathrm{~b}$, the absorbance measured at $520 \mathrm{~nm}$ is shown in the function of time passed after the homogenization of the $\mathrm{HAuCl}_{4}(0.2 \mathrm{mM}) / \mathrm{PEI}(1.2 \mathrm{mM})$ mixtures. As indicated by the graph, in the first $60 \mathrm{~min}$, the SPR peak is not detectable, whereas the absorbance grows considerably after $70 \mathrm{~min}$ due to the appearance of gold NPs (with adequate concentration and/ or size to be detected).

In the light the above-mentioned results, it is possible to affect the nucleation, growth and stabilization of the gold nanophases with the adjustment of simultaneously occurring surfactant binding. One way of doing that in a controllable manner is varying the time before the SDS is added to the
$\mathrm{HAuCl}_{4}$-PEI premix. Based on these reasoning, another solution preparation method (III method: $\mathrm{HAuCl}_{4}+\mathrm{PEI} \rightarrow$ delayed addition of SDS) was applied for a couple of compositions at surfactant excess (e.g., at 2, 4, and $8 \mathrm{mM}$ SDS), where negatively charged nanohybrids are expected to be formed according to Figure 4. This mixing protocol is an extended version of the I preparation method with one important difference; i.e. that there is a well-defined waiting period (delay time: $t_{\mathrm{D}}$ ) before the SDS solution is added to the $\mathrm{HAuCl}_{4}$-PEI premix in equal volumes (followed with rapid homogenization of the mixture).

The delay time was usually varied between 1 and $90 \mathrm{~min}$ (in some control experiments $t_{\mathrm{D}}=24 \mathrm{~h}$ and 1 week was also applied) and then the samples were stored at $25{ }^{\circ} \mathrm{C}$ before their analysis with the different experimental methods. In Figure 7, the photos and TEM images of the mixtures, prepared at $4 \mathrm{mM}$ SDS via the III method at different $t_{\mathrm{D}}$ values, are shown 1 week after the addition of the SDS solution. The delay time increases from left to right in Figure 7 (between 1 and $90 \mathrm{~min}$ ). As can be seen, the visual appearance of the samples is remarkably dependent on $t_{\mathrm{D}}$. If the SDS solution was injected and mixed with the $\mathrm{HAuCl}_{4}-\mathrm{PEI}$ premix just couple of minutes after its preparation, then the samples were bluish. With increasing delay time of SDS addition, however, the samples became purple-like, and then rosy. Similar color changes are also observable with the variation of $t_{\mathrm{D}}$ at 2 and 8 $\mathrm{mM}$ analytical SDS concentrations (see Figure S3 of the SI).

The TEM images of Figure 7 reveal interesting deviations in the morphology of the gold nanoassemblies formed at different delay times of SDS addition. At smaller $t_{\mathrm{D}}$ values, large agglomerates of AuNPs, embedded in an amorphous PEI/SDS matrix, are observable. In the case of 1 min delay time, for instance, the mean diameter of the aggregates is in the range of $40 \mathrm{~nm}$ and they are primarily composed of 4-6 crystallites with different orientations. In the sample with $t_{\mathrm{D}}=20 \mathrm{~min}$, the average diameter of the gold nanocrystals is around $20 \mathrm{~nm}$ and they contain many grain boundaries. At even larger delay times, the mean size of the gold NP agglomerates further reduces as well as they become more closed, and spherical containing mainly single crystallite grains. According to Figure S4, similar effects of $t_{\mathrm{D}}$ on the morphology of the gold nanoassemblies were found at 2 and $8 \mathrm{mM}$ SDS, with the more pronounced changes observed for the smaller surfactant concentration.

As shown in Figure 8a, the mean apparent diameter of the gold nanoassemblies reduces with increasing delay time and then it roughly levels off with a shallow minimum at $60 \mathrm{~min}$ after which the diameter values slightly increase. The minimal Stokes diameter (at around $60 \mathrm{~min}$ delay time) is commensurable with the diameter of the surfactant free PEIcapped AuNPs. The DLS data of Figure 8a also reveal that the mean size of the gold nanoassemblies prepared at larger delay times remains nearly constant over a week. Similar results were observed at 2 and $8 \mathrm{mM}$ SDS, as indicated in Figure S5a of the SI.

The changes in the mean size of the gold nanoassemblies are also reflected in the UV-vis spectra of Figure $8 b$, measured 1 week after their preparation via the III method. As indicated by the graph, with increasing delay time the intensity of the SPR peaks decreases as well as a blue shift is also observable in their position up to 50-60 min (in agreement with the reducing particle size). With a further increase of $t_{\mathrm{D}}$, however, only slight deviations in the SPR peak positions are detectable and the 


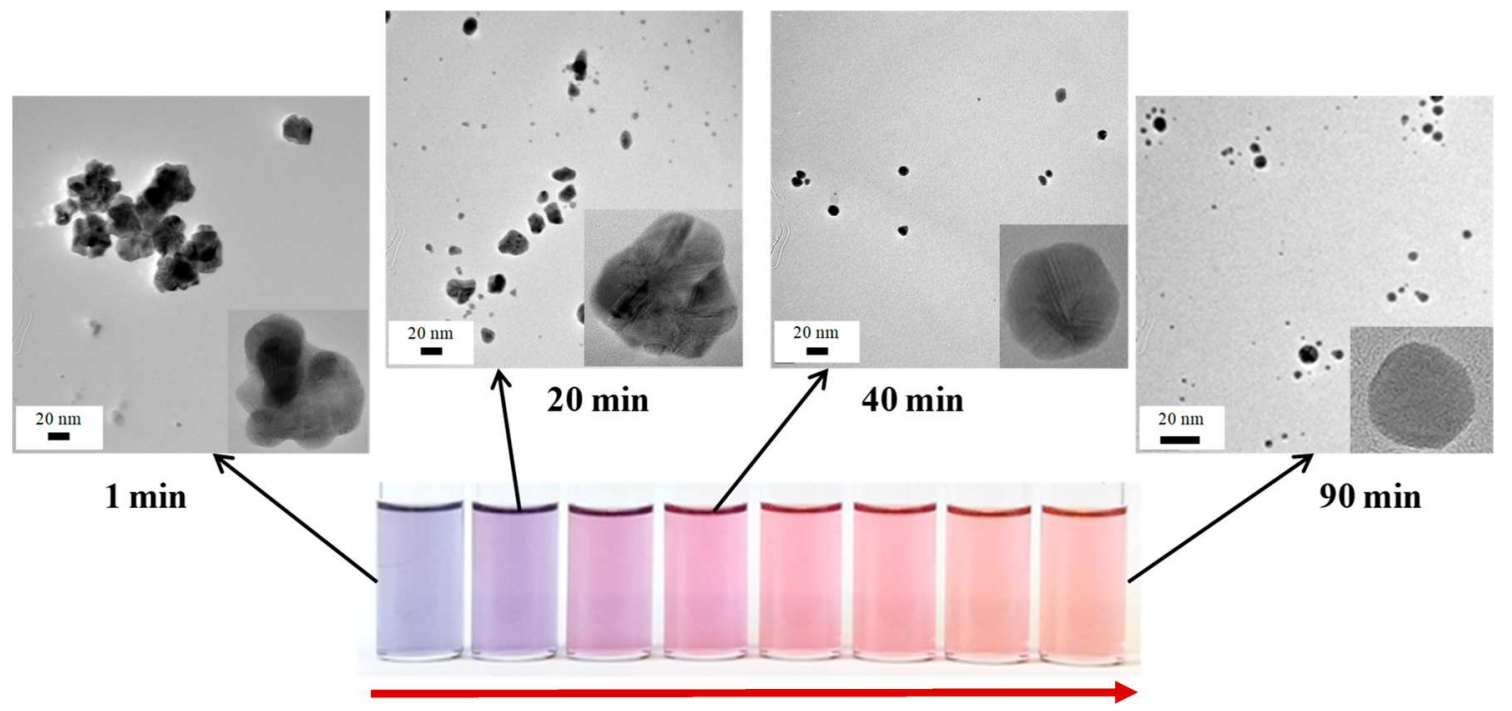

Delay time $\left(t_{D}\right)$ before SDS addition

Figure 7. Photos and TEM images of the $\mathrm{HAuCl}_{4}-\mathrm{PEI} / \mathrm{SDS}$ samples prepared by the III. method. The delay time $\left(t_{\mathrm{D}}\right)$ increases from left to right on the photo (as 1, 20, 30, 40, 50, 60, 70, and $90 \mathrm{~min}$ ). All of the samples were stored for 1 week at $25{ }^{\circ} \mathrm{C}$, before taking the pictures or the TEM images. $c_{\mathrm{SDS}}=4 \mathrm{mM}, c_{\mathrm{Au}}=0.1 \mathrm{mM}, c_{\mathrm{PEI}}=0.6 \mathrm{mM}$.

spectra closely resemble to that of the PEI capped gold NPs without SDS. The same trends in the variations of the spectra with delay time were found at 2 and $8 \mathrm{mM}$ SDS, as shown in Figures S6 and S7 of the SI.

According to Figure S5b, the electrophoretic mobility of the gold nanohybrids is not affected considerably by $t_{\mathrm{D}}$ within experimental error for the investigated SDS concentrations. This also means that the mean (electrokinetic) net charge of the formed agglomerates of nanoassemblies is not dependent largely on the timing of surfactant addition. As expected, the long-term stability of the gold NP dispersions at a given delay time increases with increasing SDS concentration due to the increasing net charge of the gold nanoassemblies (tiny amount of precipitates is only observable for the bluish systems after 1 week).

\section{POSSIBLE MECHANISM OF AU-PEI/SDS NANOASSEMBLY FORMATION}

The interpretation of the impact of the different preparation methods is far from being trivial, but it is likely to be connected to several simultaneous processes. These may include the complexation of $\mathrm{Au}(\mathrm{III})$ ions with different ligands, their reduction via amine groups, the nucleation, growth, and aggregation of gold nanophases as well as the binding and redistribution of the anionic surfactants.

In the case of the II mixing method, a considerable amount of dodecyl sulfate ion is bound onto the amine groups of PEI in the polymer/surfactant nanophases, before their mixing with $\mathrm{HAuCl}_{4}$ solution. These preformed PEI/SDS nanoparticles are small and negatively charged as indicated by their mean diameter and electrophoretic mobility data for 2,4 , and $8 \mathrm{mM}$ SDS, as shown in Table S1. Furthermore, these overcharged PEI/SDS nanophases have low $I_{1} / I_{3}$ ratio values $(\approx 1.21)$ of the pyrene fluorescent spectra, (i.e., close to the one measured for free SDS micelles $\approx 1.17$ ) due to their considerably hydrophobic interior $^{33}$ (see Table S1). In Figure 9, the time dependence of the UV-vis spectra of the SDS/PEI/HAuCl mixtures, prepared at $4 \mathrm{mM}$ SDS by the II method can be seen.
Directly after homogenization, the spectrum resemble the one measured for pure $\mathrm{HAuCl}_{4}$ solution. The subsequent changes of the spectrum in the UV range and their comparison with Figure 6 (PEI capped gold NP formation) indicate that the mixed chloro/hydroxo complex of gold(III) ions slowly transforms into $\mathrm{Au}(\mathrm{III}) /$ polyamine complex surrounded by various counterions and bound surfactant molecules. However, the preformed polymer/surfactant assemblies hinder the conversion of the mixed chloro/hydroxo complex to Au(III)/polyamine complex. On one hand, there is an electrostatic repulsion between the similarly charged PEI/SDS nanophases and that of the $\mathrm{Au}(\mathrm{III})\left(\mathrm{Cl}^{-}\right)_{x}\left(\mathrm{OH}^{-}\right)_{y}$ complexes. On the other hand, the accessibility of the amine groups of PEI within the polymer/surfactant nanoassemblies is limited due to their pronounced hydrophobic nature. ${ }^{33}$ Thus, both the reduction of $\mathrm{Au}(\mathrm{III})$ ions and the nucleation of gold NPs are hindered in this kind of environment. Furthermore, due to the gradual breakup of the PEI/SDS nanophases, the redistribution of the surfactant ions leads to irregular, large aggregates of the Au-PEI/SDS assemblies before the development of the necessary stabilizing charge of the adsorbed excess surfactant ions. This reasoning is in line with the observed slower growth of the gold nanophases and the appearance of nanoparticle agglomerates larger and more irregular than the ones formed via the application of the I preparation method. In addition, the $I_{1} / I_{3}$ ratios (see Table $S 2$ ) reveal a pronounced hydrophobic character of the gold nanoassemblies prepared via the II method.

When the PEI is mixed with $\mathrm{HAuCl}_{4}$ solution at first (I and III methods), then $\mathrm{Au}(\mathrm{III}) /$ amine complexes are formed within the hyperbranched PEI immediately as suggested in refs 20 and 25 surrounded by chloride and hydroxyl counterions. This is followed by the slow reduction of the gold(III) ions with the subsequent nucleation of the gold nanophases. Several studies also indicated that the reduction process may also result in a slight cross-linking of the ethylenimine groups. ${ }^{20}$ Therefore, the fusion of the primary gold nanophases leads to AuNPs entrapped within the PEI molecules ${ }^{25}$ and stabilized 
a.)

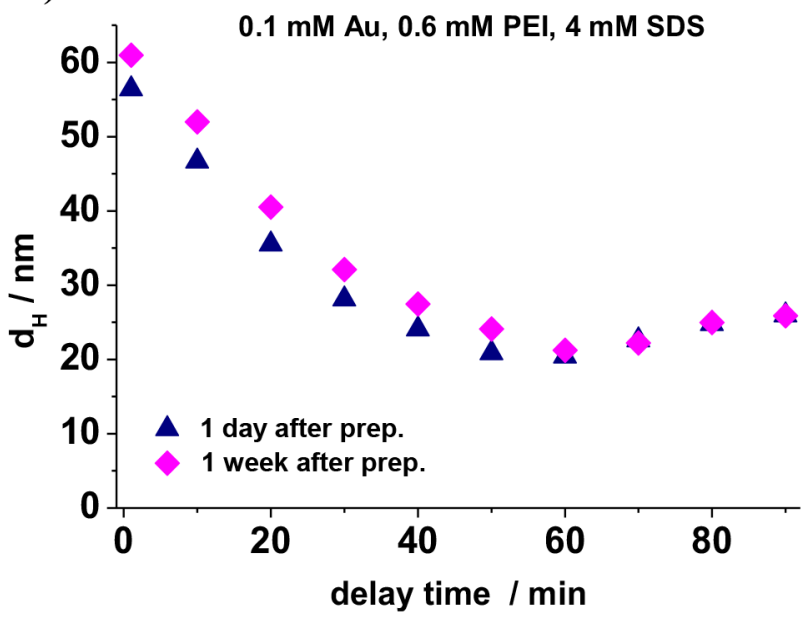

b.)

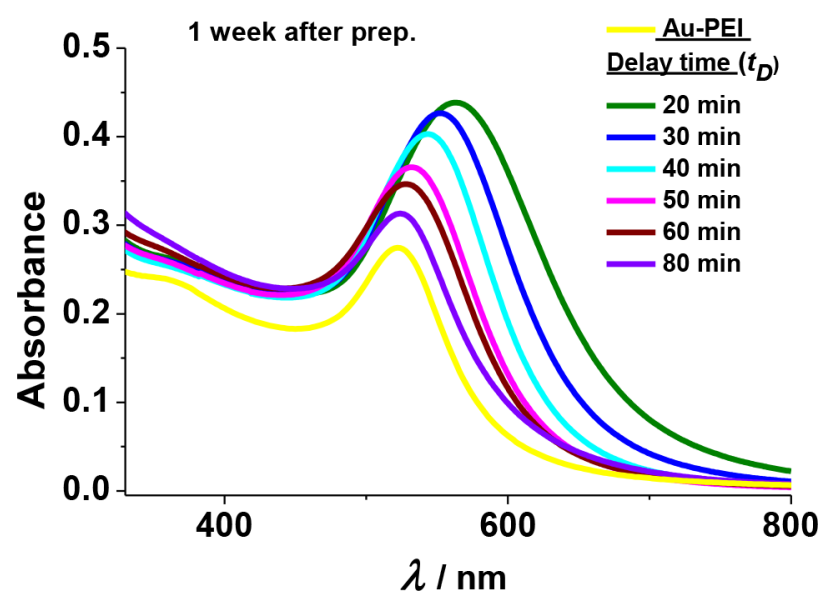

Figure 8. (a) Apparent mean diameter of the gold nanoassemblies against the delay time before SDS addition $\left(t_{\mathrm{D}}\right)$. The data were measured 1 day and 1 week after the preparation of the mixtures by the III method. (b) UV-vis spectra of the samples with different $t_{\mathrm{D}}$ values, 1 week after their preparation. The size of the symbols, and the width of the lines are commensurable with the standard error of the measurements. $c_{\mathrm{SDS}}=4 \mathrm{mM}, c_{\mathrm{Au}}=0.1 \mathrm{mM}$, and $c_{\mathrm{PEI}}=0.6 \mathrm{mM}$.

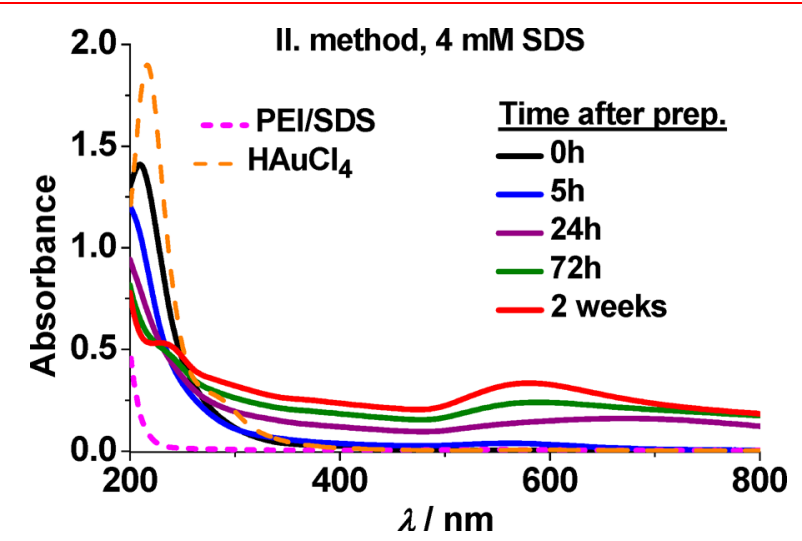

Figure 9. Time dependence of the SDS/PEI/HAuCl ${ }_{4}$ mixtures after their preparation by the II method at $25^{\circ} \mathrm{C}$. The width of the lines is commensurable with the standard error of the measurements. $c_{\mathrm{SDS}}=4$ $\mathrm{mM}, c_{\mathrm{Au}}=0.1 \mathrm{mM}$, and $c_{\mathrm{PEI}}=0.6 \mathrm{mM}$. through the charges of the remaining protonated amine groups at $\mathrm{pH}=4$.

The impact of SDS addition clearly depends on the actual stage of the reduction and nucleation processes within the PEI molecules. Figure 8a reveals that the most prominent changes in the size of the mixed gold nanoassemblies upon SDS addition occur in the 1-60 min delay time range. One may speculate, therefore, that if $t_{\mathrm{D}}$ is higher than $60 \mathrm{~min}$, then the reduction process leads to nanoassemblies with considerable amount of gold nucleuses already embedded within the PEI matrix. The gold NP nucleation also leads to more free amine groups, (not occupied further in the gold(III)/amine complexes) available for SDS binding. After SDS addition, the surfactant ions bind instantaneously to the remaining amine groups of PEI forming a hydrophobic neutral shell around the core, where further growth and/or fusion of the gold nanophases could occur within the PEI molecules. This is followed by the fast adsorption of dodecyl sulfate ions on the surface of this hydrophobic shell, creating an appropriate negative charge necessary for the stabilization of these hierarchical assemblies (similarly to the stabilization of overcharged PEI/SDS particles ${ }^{33-35}$ ).

In Figure 10a, the variation of the UV-vis spectra of $\mathrm{Au}$ PEI/SDS mixtures with time is shown after the addition of SDS at $t_{\mathrm{D}}=90 \mathrm{~min}$. The graph indicates that the mixed gold nanoassemblies evolve similarly compared to the development of pure PEI-capped gold NPs (as shown in Figure 6 and S2). This was further supported by the comparison with control experiments, where the $\mathrm{HAuCl}_{4}$-PEI premix was mixed with water in equal volumes (instead of SDS solution) after $t_{\mathrm{D}}=90$ min delay time. In Figure 10b, the spectra of these surfactant free $\mathrm{HAuCl}_{4}$-PEI mixture are shown immediately and $48 \mathrm{~h}$ after the dilution with water together with the corresponding spectra of $\mathrm{HAuCl}_{4}-\mathrm{PEI} / \mathrm{SDS}$ system (prepared $t_{\mathrm{D}}=90 \mathrm{~min}$ ). The comparison of the spectra clearly reveals that the time scale of gold nanoassembly formation is very similar in both cases. This also means that the addition of SDS to the $\mathrm{HAuCl}_{4}$-PEI premix at larger delay times (i.e., $t_{\mathrm{D}}>60 \mathrm{~min}$ ) does not cause significant aggregation. This reasoning is also in agreement with the time independent small mean diameters of Au-PEI/SDS NPs detected with $t_{\mathrm{D}}=60(22 \mathrm{~nm})$ and $90 \mathrm{~min}$ $(26 \mathrm{~nm})$ measured immediately after the addition of SDS up to 1 week.

However, if the delay time is much less than $60 \mathrm{~min}$ (within a couple of minutes for instance) then the surfactant addition may have different consequences than the ones discussed above. First of all, a considerable part of the ethylenimine groups of PEI is involved in $\mathrm{Au}(\mathrm{III}) /$ amine complexes (possibly in planar arrangements locally with four amine groups per gold(III) ions), thus the surfactant ions could bind only to the available protonated amine groups. In addition, the dodecyl sulfate ions partly replace the chloride and hydroxyl counterions of the gold(III)/amine complexes. (This counterion effect may be behind the appearance of a small local minimum in the UV range. ${ }^{44}$ ) Due to the hydrophobic environment of the surrounding PEI/SDS assemblies and their impact on the optimal structure of the local $\mathrm{Au}(\mathrm{III}) /$ amine complexes, the reduction process as well as the fusion of the gold nucleuses could be considerably hindered. This, in turn, may slow down the nucleation of the gold nanophases and the development of a stabilizing surfactant adsorption layer resulting in considerably larger NPs compared to the surfactant free PEI-capped gold NPs (and the ones formed at larger $t_{\mathrm{D}}$ 
a.)

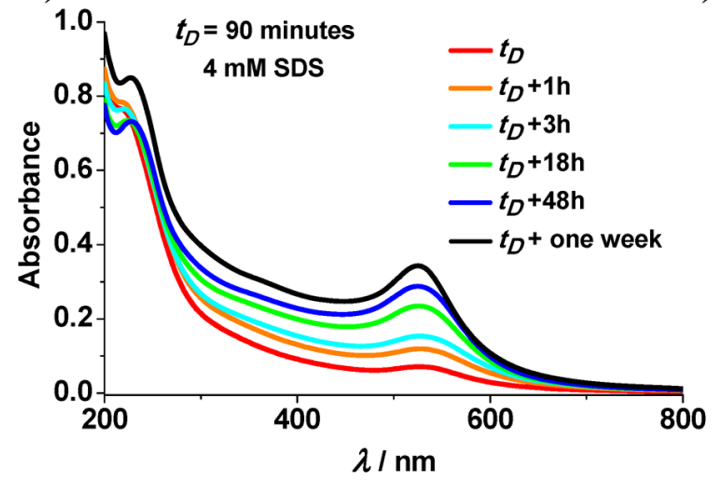

b.)

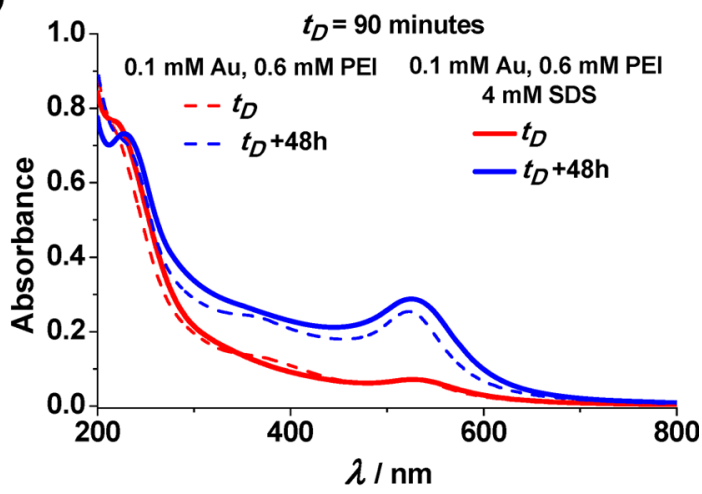

c.)

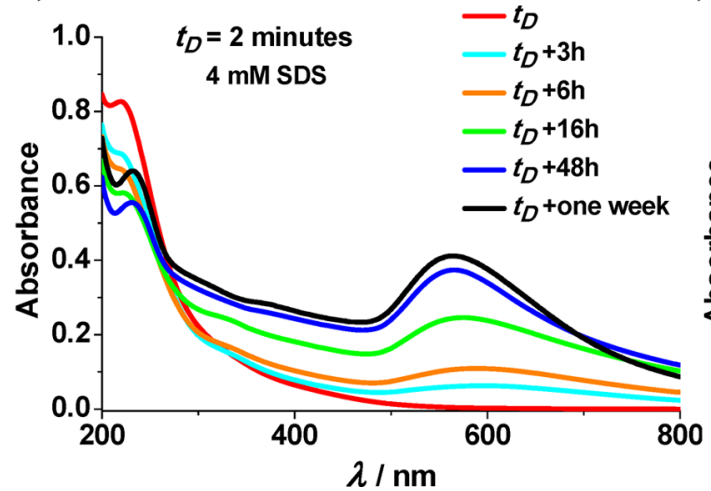

d.)

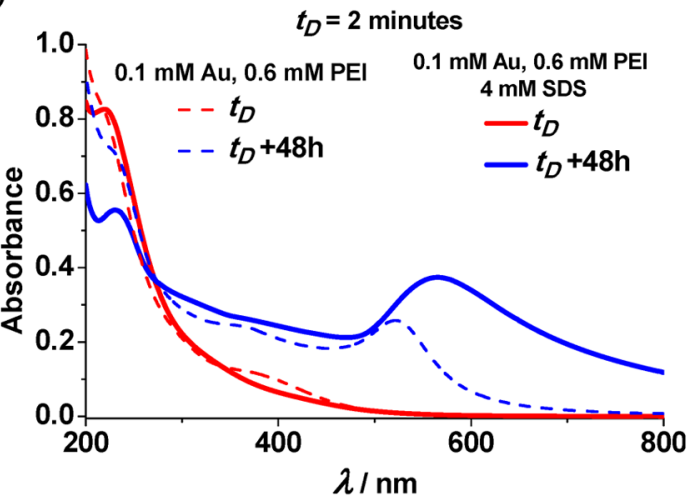

Figure 10. Time dependence of the $\mathrm{HAuCl}_{4}-\mathrm{PEI} / \mathrm{SDS}$ mixtures after their preparation by the III method (a) at $t_{\mathrm{D}}=90$ and (c) 2 min delay times. UV-vis spectra of the samples measured immediately (red solid lines) and $48 \mathrm{~h}$ (blue solid lines) after the addition of surfactant ( $8 \mathrm{mM}$ SDS) to the $\mathrm{HAuCl}_{4}-\mathrm{PEI}$ premix $\left(c_{\mathrm{Au}}=0.2 \mathrm{mM}\right.$ and $\left.c_{\mathrm{PEI}}=1.2 \mathrm{mM}\right)(\mathrm{b})$ at $t_{\mathrm{D}}=90$ and (d) 2 min delay times. For the sake of comparison, control experiments were carried out where the $\mathrm{HAuCl}_{4}-\mathrm{PEI}$ premix (with $c_{\mathrm{Au}}=0.2 \mathrm{mM}$ and $c_{\mathrm{PEI}}=1.2 \mathrm{mM}$ ) was mixed with water in equal volumes after $t_{\mathrm{D}}=2$ and 90 min delay times. The corresponding spectra of these half diluted $\mathrm{HAuCl}_{4}-\mathrm{PEI}$ mixtures were measured immediately (red dashed lines) and $48 \mathrm{~h}$ (blue dashed lines) after the addition of water and shown in Figure 10b and d. The width of the lines is commensurate with the standard error of the measurements. Final concentrations: $c_{\mathrm{SDS}}=4 \mathrm{mM}$ or $0 \mathrm{mM}, c_{\mathrm{Au}}=0.1 \mathrm{mM}$, and $c_{\mathrm{PEI}}=0.6 \mathrm{mM}$.

values). This reasoning is in line with the data of Figure 10c, where the time dependence of the UV-vis spectra of $\mathrm{HAuCl}_{4}-\mathrm{PEI} / \mathrm{SDS}$ mixtures, prepared with $t_{\mathrm{D}}=2 \mathrm{~min}$, is shown. The formation of large gold agglomerates with less pronounced SPR peak is also indicated in Figure 10d, where the evolution of surfactant free PEI capped gold NPs is compared with the corresponding Au-PEI/SDS nanoassemblies after the addition of water or SDS to the $\mathrm{HAuCl}_{4}-\mathrm{PEI}$ premix at $t_{\mathrm{D}}=2 \mathrm{~min}$.

These findings are attributable to the fact that in the beginning of the gold nucleation process the binding sites of dodecyl sulfate ions is limited due to the formation gold(III)/ amine complexes. Thus, the driving force for the adsorption of excess surfactant ions, i.e. for the development of overcharged hydrophobic PEI/SDS layers around the gold NPs, is low. Therefore, aggregation of the mixed gold nanoassemblies into larger agglomerates occurs. As the reduction process of gold(III) ions proceeds, however, more and more amine groups become available for SDS binding. This leads to the development of a more hydrophobic PEI/SDS layer in the outer region of these gold NP agglomerates and their stabilization with the adsorption of excess dodecyl sulfate ions.

This also suggests that a slow reorganization of the surfactant ions could occur during the gold NP nucleation and growth. This process is manifested in the time dependence of the pyrene fluorescence results, shown in Figure 11. As

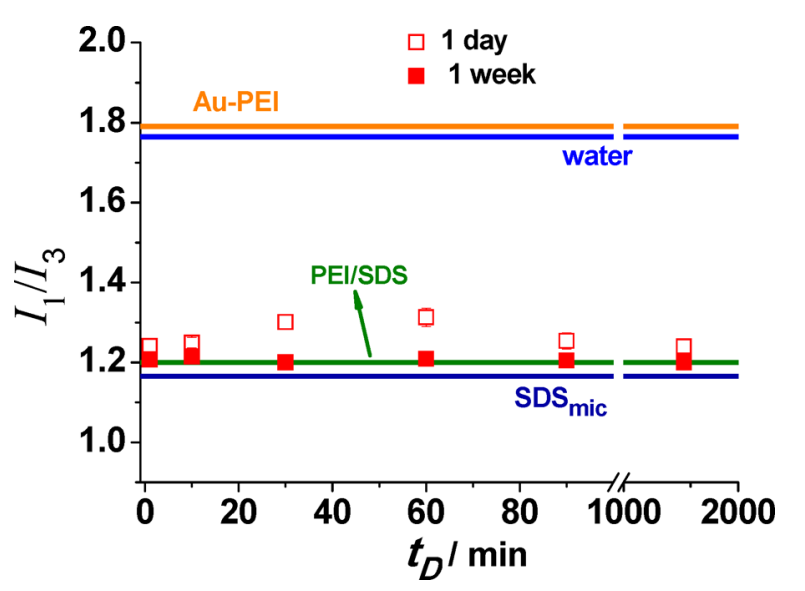

Figure 11. $I_{1} / I_{3}$ ratios of the $\mathrm{HAuCl}_{4}-\mathrm{PEI} / \mathrm{SDS}$ mixtures as a function of delay time before SDS addition. The pyrene was added 1 day (open square) or 1 week (solid square) after the preparation of the system at a given $t_{\mathrm{D}}$ and then the mixtures were stirred for 1 day in order to solubilize completely the pyrene molecules.

indicated by the graph and the data of Table S2, the $I_{1} / I_{3}$ ratios of pure PEI/SDS samples and that of the Au-PEI/SDS assemblies prepared by the II method or via the III method with very small or much larger delay times (i.e., $t_{\mathrm{D}}<10$ and $t_{\mathrm{D}}$ $>90 \mathrm{~min}$ ) are similar and close to the value measured for the 
hydrophobic interior of free SDS micelles. Furthermore, the observed $I_{1} / I_{3}$ values of these samples are not dependent on the time passed after the preparation of the systems within 1 week.

The most remarkable feature of Figure 11 is that at intermediate delay times $\left(10 \mathrm{~min}<t_{\mathrm{D}}<90 \mathrm{~min}\right)$ larger $I_{1} / I_{3}$ ratios and a maximum in the function of $t_{\mathrm{D}}$ were observed, provided that the pyrene was added to the mixtures 1 day after their preparation. However, if the pyrene was solubilized in the systems 1 week after their preparation, then all the measured $I_{1} / I_{3}$ ratios become indistinguishable within experimental error and equal to the one found for pure (gold free) PEI/SDS assemblies $(\approx 1.21)$. This finding might be explained qualitatively by the gradual redistribution and organization of the bound and unbound surfactant ions, which is expected to occur at intermediate delay times, when the reduction process has already started but it is still far from being completed. In this case, the distribution of the surfactant binding sites on PEI is not ideal for the formation of regular polymer/surfactant assemblies, which are accessible for pyrene solubilization. However, as the reduction proceeds further and more amine groups become free than the extra surfactant binding can create more accessible and/or larger PEI/SDS assemblies with pronounced hydrophobic interior and lower $I_{1} / I_{3}$ ratio.

\section{CONCLUSIONS}

In the present paper, we have shown that the PEI-assisted gold nanoparticle formation could be tuned at $25{ }^{\circ} \mathrm{C}$ via the controlled order and timing of SDS addition. At this temperature the reduction of the $\mathrm{Au}$ (III) ions via the amine groups as well as the nucleation of the gold NPs is much slower than at elevated temperatures, and they may occur simultaneously with the surfactant binding on the amine groups of the polymer. Therefore, both the order of addition of the surfactant and the time passed before its addition (delay time) could play a crucial role in the properties of the formed gold nanoassemblies.

If the surfactant is added at large excess to the $\mathrm{HAuCl}_{4}-\mathrm{PEI}$ premix, then the properties of the formed overcharged gold nanoassemblies are largely dependent on the delay time before SDS addition. Specifically, we have shown that via varying the delay time of surfactant addition between 1 and $90 \mathrm{~min}$, the size and shape of the gold assemblies can be tuned simply and reproducibly. Furthermore, the timing of surfactant addition also affects the morphology of the formed gold NPs, due to the formation of hydrophobic PEI/SDS reservoirs in the vicinity of the preformed gold nucleuses and gold(III)/amine complexes.

In contrast, when $\mathrm{HAuCl}_{4}$ is added to the PEI/SDS mixtures, then the accessibility of the amine groups for the $\mathrm{Au}$ (III) ions is considerably hindered due to the hydrophobic internal structure of the preformed PEI/SDS assemblies. Thus, the gold NP formation slows down and the stabilizing effect of the excess adsorbed surfactant ions is also attenuated. This results in the formation of large gold aggregates and/or precipitates over the whole investigated composition range of surfactant excess.

The present study clearly demonstrated that the manipulation of the simultaneous $\mathrm{Au}$ (III)/amine complex formation, gold NP nucleation and surfactant binding processes at room temperature largely affects the size distribution, hydrophobicity and morphology of the PEI entrapped gold NPs. The results may be further extended for versatile applications, which are based of noble metal nanohybrids with controlled size, charge, and hydrophobic environment.

\section{ASSOCIATED CONTENT}

\section{Supporting Information}

The Supporting Information is available free of charge on the ACS Publications website at DOI: 10.1021/acs.langmuir.9b03055.

Additional experimental information and graphs as discussed in the text (PDF)

\section{AUTHOR INFORMATION}

\section{Corresponding Author}

*Phone: +36-1-372-2500/6238. E-mail: meszaros@chem.elte. hu.

\section{ORCID}

Brigitta Dúzs: 0000-0002-0909-2808

Róbert Mészáros: 0000-0001-9638-2207

Notes

The authors declare no competing financial interest.

\section{ACKNOWLEDGMENTS}

This work was completed in the ELTE Institutional Excellence Program (1783-3/2018/FEKUTSRAT) supported by the Hungarian Ministry of Human Capacities. This publication is also the partial result of the Research \& Development Operational Programme for the project "Modernization and Improvement of Technical Infrastructure for Research and Development of J. Selye University in the Fields of Nanotechnology and Intelligent Space” ITMS 26210120042 and of the Research \& Innovation Operational Programme for the project: "Support of Research and Scientific Capacities in the Fields of Nanochemistry and Supramolecular Systems", ITMS NFP313010T583, cofunded by the European Regional Development Fund. G.S. and B.P. thank the microscope infrastructural project, Grant No. VEKOP-2.3.3-15-201600002, for support.

\section{REFERENCES}

(1) Yang, P.; Zheng, J.; Xu, Y.; Zhang, Q.; Jiang, L. Colloidal Synthesis and Applications of Plasmonic Metal Nanoparticles. Adv. Mater. 2016, 28, 10508-10517.

(2) Daniel, M.-C.; Astruc, D. Gold Nanoparticles: Assembly, SupramolecularChemistry, Quantum-Size-RelatedProperties, and ApplicationstowardBiology, Catalysis, and Nanotechnology. Chem. Rev. 2004, 104, 293-346.

(3) Sengani, M.; Grumezescu, A. M.; Rajeswari, V. D. Recent Trends and Methodologies in Gold Nanoparticle Synthesis - A Prospective Review on Drug Delivery Aspect. OpenNano 2017, 2, 37-46.

(4) Zhu, J.; Wang, G.; Alves, C. S.; Tomás, H.; Xiong, Z.; Shen, M.; Rodrigues, J.; Shi, X. Multifunctional Dendrimer-Entrapped Gold Nanoparticles Conjugated with Doxorubicin for $\mathrm{pH}$-Responsive Drug Delivery and Targeted Computed Tomography Imaging. Langmuir 2018, 34, 12428-12435.

(5) Grzelczak, M.; Pérez-Juste, J.; Mulvaney, P.; Liz-Marzán, L. M. Shape control in gold nanoparticle synthesis. Chem. Soc. Rev. 2008, $37,1783-1791$.

(6) Alex, S.; Tiwari, A. Functionalized Gold Nanoparticles: Synthesis, Properties and Applications-A Review. J. Nanosci. Nanotechnol. 2015, 15, 1869-1894.

(7) Vaseghi, Z.; Nematollahzadeh, A.; Tavakoli, O. Green Methods for the Synthesis of Metal Nanoparticles Using Biogenic Reducing Agents: A Review. Rev. Chem. Eng. 2018, 34, 529-559. 
(8) Gittins, D. I.; Caruso, F. Tailoring the Polyelectrolyte Coating of Metal Nanoparticles. J. Phys. Chem. B 2001, 105, 6846-6852.

(9) Mayya, K. S.; Schoeler, B.; Caruso, F. Preparation and Organization of Nanoscale Polyelectrolyte-Coated Gold Nanoparticles. Adv. Funct. Mater. 2003, 13, 183-188.

(10) Harris, C. M.; Miller, S. G.; Andresen, K.; Thompson, L. B. Quantitative Measurement of Sodium Polystyrene Sulfonate Adsorption onto CTAB Capped Gold Nanoparticles Reveals Hard and Soft Coronas. J. Colloid Interface Sci. 2018, 510, 39-44.

(11) Fuller, M.; Köper, I. Polyelectrolyte-Coated Gold Nanoparticles: The Effect of Salt and Polyelectrolyte Concentration on Colloidal Stability. Polymers 2018, 10, 1336.

(12) Nayak, S.; Fieg, M.; Wang, W.; Bu, W.; Mallapragada, S.; Vaknin, D. Effect of (Poly)electrolytes on the Interfacial Assembly of Poly(ethylene glycol)-Functionalized Gold Nanoparticles. Langmuir 2019, 35, 2251-2260.

(13) Sun, X.; Dong, S.; Wang, E. One-Step Synthesis and Characterization of Polyelectrolyte-Protected Gold Nanoparticles through a Thermal Process. Polymer 2004, 45, 2181-2184.

(14) Sun, X.; Dong, S.; Wang, E. High-Yield Synthesis of Large Single-Crystalline Gold Nanoplates through a Polyamine Process. Langmuir 2005, 21, 4710-4712.

(15) Yuan, Z.; Du, Y.; He, Y. Hyperbranched Polyamine Assisted Synthesis of Dual-Luminescent Gold Composite with $\mathrm{pH}$ Responsive Character. Methods Appl. Fluoresc. 2017, 5, 014011.

(16) Sun, X.; Dong, S.; Wang, E. One-Step Preparation of Highly Concentrated Well-Stable Gold Colloids by Direct Mix of Polyelectrolyte and $\mathrm{HAuCl}_{4}$ Aqueous Solutions at Room Temperature. J. Colloid Interface Sci. 2005, 288, 301-303.

(17) Wang, S. T.; Yan, J. C.; Chen, L. Formation of Gold Nanoparticles and Self-Assembly Into Dimer and Trimer Aggregates. Mater. Lett. 2005, 59, 1383-1386.

(18) Yu, T.; Kim, R.; Park, H.; Yi, J.; Kim, W.-S. Mechanistic Study of Synthesis of Gold Nanoparticles Using Multi-Functional Polymer. Chem. Phys. Lett. 2014, 592, 265-271.

(19) Sun, X.; Dong, S.; Wang, E. One-Step Polyelectrolyte-Based Route to Well-Dispersed Gold Nanoparticles: Synthesis and Insight. Mater. Chem. Phys. 2006, 96, 29-33.

(20) Kretschmer, F.; Mansfeld, U.; Hoeppener, S.; Hager, M. D.; Schubert, U. S. Tunable Synthesis of Poly(Ethylene Imine)-Gold Nanoparticle Clusters. Chem. Commun. 2014, 50, 88-90.

(21) Mikami, T.; Takayasu, Y.; Hirasawa, I. PEI-Assisted Preparation of Au Nanoparticles via Reductive Crystallization Process. Chem. Eng. Res. Des. 2010, 88, 1248-1251.

(22) Mikami, T.; Takayasu, Y.; Watanabe, J.; Hirasawa, I. Influence of Polyethyleneimine Addition on Crystal Size Distribution of Au Nanocrystals. Chem. Eng. Technol. 2011, 34, 583-586.

(23) Lazarus, G. G.; Singh, M. In Vitro Cytotoxic Activity and Transfection Efficiency of Polyethyleneimine Functionalized Gold Nanoparticles. Colloids Surf., B 2016, 145, 906-911.

(24) Zhou, B.; Xiong, Z.; Wang, P.; Peng, C.; Shen, M.; Shi, X. Acetylated Polyethylenimine-Entrapped Gold Nanoparticles Enable Negative Computed Tomography Imaging of Orthotopic Hepatic Carcinoma. Langmuir 2018, 34, 8701-8707.

(25) Mulens-Arias, V.; Nicolás-Boluda, A.; Gehanno, A.; Balfourier, A.; Carn, F.; Gazeau, F. Polyethyleneimine-Assisted One-Pot Synthesis of Quasi-Fractal Plasmonic Gold Nanocomposites as a PhotothermalTheranostic Agent. Nanoscale 2019, 11, 3344-3359.

(26) Philip, A.; Ankudze, B.; Pakkanen, T. T. PolyethylenimineAssistedSeed-MediatedSynthesis of Gold NanoparticlesforSurfaceEnhancedRamanScatteringStudies. Appl. Surf. Sci. 2018, 444, 243252.

(27) Wen, S.; Zheng, F.; Shen, M.; Shi, X. Synthesis of Polyethyleneimine-Stabilized Gold NanoparticlesforColorimetricSensing of Heparin. Colloids Surf., A 2013, 419, 80-86.

(28) Kim, I.-H.; Kim, J. H.; Choi, J.-Y.; Shin, C. H.; Kim, J.-H.; Bae, G.-T.; Shin, K. S. Tuning the Interparticle Distances in SelfAssembled Gold Nanoparticle Films with Their Plasmonic Responses. Chem. Phys. Lett. 2019, 715, 91-99.
(29) Sánchez-Iglesias, A.; Grzelczak, M.; Altantzis, T.; Goris, B.; Pérez-Juste, J.; Bals, S.; Van Tendeloo, G.; Donaldson, S. H.; Chmelka, B. F., Jr.; Israelachvili, J. N.; Liz-Marzán, L. M. HydrophobicInteractionsModulateSelf-assembly of Nanoparticles. ACS Nano 2012, 6, 11059-11065.

(30) Xi, C.; Marina, P. F.; Xia, H.; Wang, D. DirectedSelf-Assembly of Gold Nanoparticles Into Plasmonic Chains. Soft Matter 2015, 11, $4562-4571$.

(31) Mitamura, K.; Imae, T.; Saito, N.; Takai, O. Fabrication and Self-Assembly of Hydrophobic Gold Nanorods. J. Phys. Chem. B 2007, 111, 8891-8898.

(32) Bali, K.; Sáfrán, G.; Pécz, B.; Mészáros, R. Preparation of Gold Nanocomposites with Tunable Charge and Hydrophobicity via the Application of Polymer/Surfactant Complexation. ACS Omega 2017, 2, 8709-8716.

(33) Mészáros, R.; Thompson, L.; Bos, M.; Varga, I.; Gilányi, T. Interaction of Sodium Dodecyl Sulfate with Polyethyleneimine: Surfactant-Induced Polymer Solution Colloid Dispersion Transition. Langmuir 2003, 19, 609-615.

(34) Mezei, A.; Mészáros, R. Novel Nanocomplexes of Hyperbranched Poly(Ethyleneimine), Sodium Dodecyl Sulfate and Dodecyl Maltoside. Soft Matter 2008, 4, 586-592.

(35) Mezei, A.; Mészáros, R.; Varga, I.; Gilányi, T. Effect of Mixing on the Formation of Complexes of Hyperbranched Cationic Polyelectrolytes and Anionic Surfactants. Langmuir 2007, 23, 42374247.

(36) Bastardo, L.; Garamus, V. M.; Bergström, M.; Claesson, P. M. The Structures of Complexes between Polyethylene Imine and Sodium Dodecyl Sulfate in D2O: A Scattering Study. J. Phys. Chem. B 2005, 109, 167-174.

(37) Zhou, S.; Burger, C.; Chu, B. Supramolecular Structures of Polyethylenimine-Sodium Alkyl Sulfate Complexes. J. Phys. Chem. B 2004, 108, 10819-10824.

(38) Varga, I.; Mezei, A.; Mészáros, R.; Claesson, P. M. Controlling the Interaction of Poly(Ethylene Imine) Adsorption Layers with Oppositely Charged Surfactant by Tuning the Structure of the Preadsorbed Polyelectrolyte Layer. Soft Matter 2011, 7, 1070110712.

(39) Naderi, A.; Claesson, P. M.; Bergström, M.; Dédinaitè, A. Trapped Non-Equilibrium States in Aqueous Solutions of Oppositely Charged Polyelectrolytes and Surfactants: Effects of Mixing Protocol and Salt Concentration. Colloids Surf., A 2005, 253, 83-93.

(40) Fegyver, E.; Mészáros, R. The Impact of Nonionic Surfactant Additives on the Nonequilibrium Association Between Oppositely Charged Polyelectrolytes and Ionic Surfactants. Soft Matter 2014, 10, 1953-1962.

(41) Bali, K.; Varga, Z.; Kardos, A.; Varga, I.; Gilányi, T.; Domján, A.; Wacha, A.; Bóta, A.; Mihály, J.; Mészáros, R. Effect of Dilution on the Nonequilibrium Polyelectrolyte/Surfactant Association. Langmuir 2018, 34, 14652-14660.

(42) Llamas, S.; Guzmán, E.; Akanno, A.; Fernández-Peña, L.; Ortega, F.; Campbell, R. A.; Miller, R.; Rubio, R. G. Study of the Liquid/Vapor Interfacial Properties of Concentrated PolyelectrolyteSurfactant Mixtures Using Surface Tensiometry and Neutron Reflectometry: Equilibrium, Adsorption Kinetics, and Dilational Rheology. J. Phys. Chem. C 2018, 122, 4419-4427.

(43) Wang, S.; Qian, K.; Bi, X.; Huang, W. Influence of Speciation of Aqueous $\mathrm{HAuCl}_{4}$ on the Synthesis, Structure, and Property of $\mathrm{Au}$ Colloids. J. Phys. Chem. C 2009, 113, 6505-6510.

(44) Gangopadhayay, A. K.; Chakravorty, A. Charge Transfer Spectra of some Gold(III) Complexes. J. Chem. Phys. 1961, 35, 2206-2209. 